\title{
Alternate Rapid Maxillary Expansion and Constriction (Alt-RAMEC) protocol: A Comprehensive Literature Review
}

\author{
Muhammed Hilmi Büyükçavuş (D)
}

Private Practice, Afyonkarahisar, Turkey

Cite this article as: Büyükçavuş MH. Alternate Rapid Maxillary Expansion and Constriction (Alt-RAMEC) protocol: A Comprehensive Literature Review. Turk J Orthod 2019; 32(1): 47-51.

\section{ABSTRACT}

\begin{abstract}
The aim of this comprehensive review is to introduce clinicians to the increasingly popularity Alt-RAMEC procedure, a method commonly used in the treatment of class III malocclusion in the last 15 years. Another application of the literature to enhance the skeletal effects of Class III treatment on the maxillae is the Alternative Rapid Maxillary Expansion and Constriction (Alt-RAMEC) procedure introduced by Liou, which improves the effectiveness of the maxillae relative to the surrounding sutures and the enhancement of the maxillae. In the Alt-RAMEC protocol, maxillae will be enlarged to be $1 \mathrm{~mm}$ per day, first enlarged to $7 \mathrm{~mm}$, and then the $1 \mathrm{~mm}$ screw is closed. In other weeks, in this order the screw of the expansion device is turned on for one week and then closed for one week, completing the Alt-RAMEC protocol at the end of the 9-week process. In this review, we will discuss the advantages and disadvantages of the studies, which include successful treatments by applying this protocol, differences with other methods, its effect on the airway, and its advantages and disadvantages.
\end{abstract}

Keywords: Alt-RAMEC, Class III malocclusion, literature review

\section{INTRODUCTION}

For centuries, Class III malocclusions have attracted more attention than other problems in orthodontics (1). Even in Renaissance portraits and paintings, Class III malocclusions appear to be a significant feature (2). Today, patients are even more aware of the apparent effects on the external appearance, and therefore even less frequent than other malocclusions, due to their adverse effects on the psychosocial status of the patients. These malocclusions, which are primarily etiologically caused by genetics, involve mandibular prognathism, maxillary retrognathism, or a combination of these two conditions (2-3).

There are two approaches of the treatment of Class III anomalies according to patient status. Among these approaches, orthopedic treatments are used to treat patients in the growth-development period, and the other approach is camouflage treatment or orthognathic surgical treatment, which is preferred in adult individuals whose growth-development has been completed (4).

In cases of growth-development period, mandibular treatment is used to prevent and direct the development of mandibles in cases of mandibular origin with chin cap, and maxillary protraction treatments are performed with frequent face mask in cases of maxilla origin. In the literature, we found that there are some researchers who utilized the face mask application in combination with several methods in maxillary protraction (5).

The purpose of this method is to increase the efficiency of an appliance that requires patient cooperation, to perform more prototypes in a short period, and to reduce unwanted dentoalveolar effects and obtain more skel- 
etal effects. Studies have shown that increasing the skeletal effect can reduce post-treatment relapse, which is one of the most significant problems in orthodontic treatment (6-8). In light of this information, researchers have implemented face masks with the rapid maxillary expansion (RME) appliance (9-10), skeletal anchorage (11), and Alternate Rapid Maxillary Expansion and Constriction (Alt-RAMEC) protocol (12-13) to increase the skeletal efficacy in the procedure.

\section{Alt-RAMEC Protocol}

In 2005, the Alt-RAMEC protocol was introduced by Liou; it enables sutural mobilization with the opening and closing of the RME screw for 7-9 consecutive weeks without unnecessary expansion (14). Its rationale is similar to that of simple tooth extraction in which we repeatedly rock the tooth buccally and lingually until the tooth is "disarticulated" out of the alveolar socket (12).

First, RME performed prototyping by providing sutural activation with the device. Some researchers report that a $5 \mathrm{~mm}$ expanse is sufficient for sutural mobilization, whereas other researchers state that at least 12-15 mm expansions are needed. However, such an excessive expansion may cause clinical irritation of the palatal mucosa, as well as a marked discrepancy between the maxillary and the mandible in non-restrictive individuals (15).
In the Alt-RAMEC protocol, the maxilla is expanded by $7 \mathrm{~mm}$ on week 1 through an expansion device that expands $1 \mathrm{~mm} /$ day, and then the screw is closed at a rate of $1 \mathrm{~mm} / \mathrm{s}$ on week 2 . In the remaining weeks, the screw of the expansion device is turned on for 1 week and closed for 1 week, and the Alt-RAMEC protocol is completed at the end of the 9-week cycle. Following completion of this protocol, protraction force is applied to move the maxillae forward (12).

In his article introducing the Liou Alt-RAMEC protocol, Liou schematized the changes that occur in the maxillae with RME and Alt-RAMEC (Figure 1). With the hyrax-type RME, the center of rotation of the maxillae is opened at the point of the PNS, and the tuber maxillae move forward and backward as the A point is located at the same position according to the amount of resorption in the maxilla. With the Alt-RAMEC, the center of rotation of the maxillae is opened at the point of the PNS, and the tuber maxillae move forward more without any resorption in the maxilla. Owing to this protocol, there is much more mobilization in the sutures, and resistance structures (e.g., sphenoid) are weakened (12).

\section{Comparison of Alt-RAMEC Protocol and RME}

Face mask application after the 1-week RME and face mask therapy after the Alt-RAMEC protocol in different procedures were compared in the majority of studies involving the Alt-RAMEC a

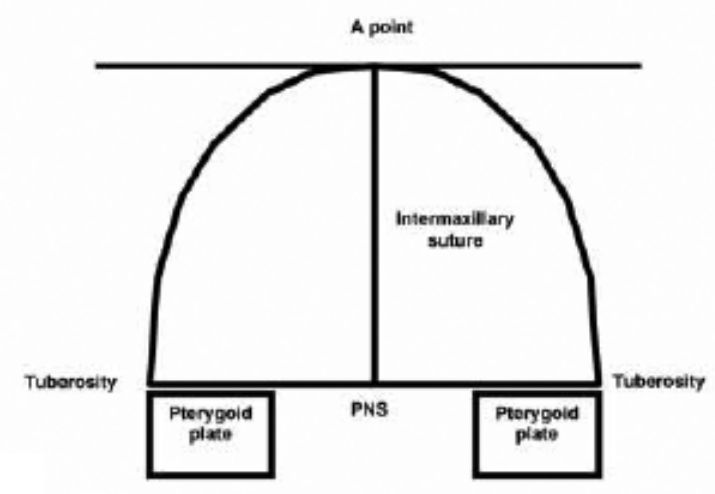

C

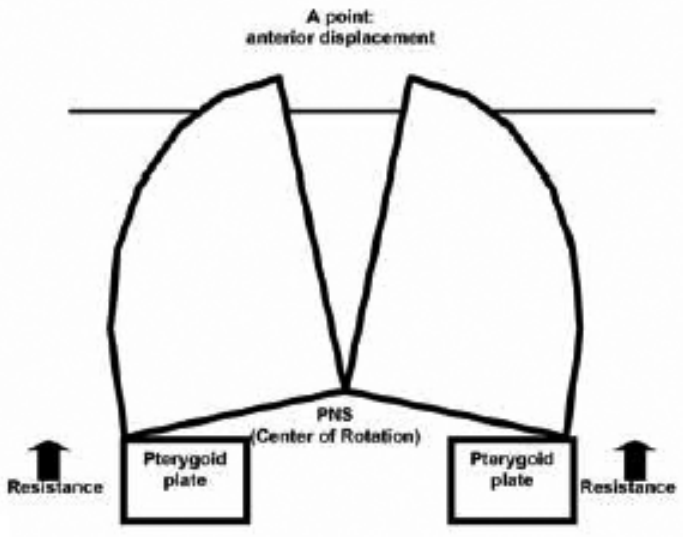

b

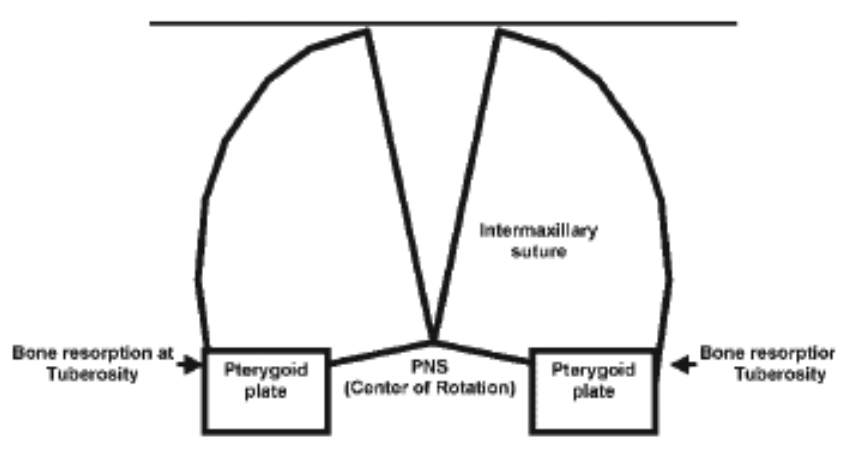

d

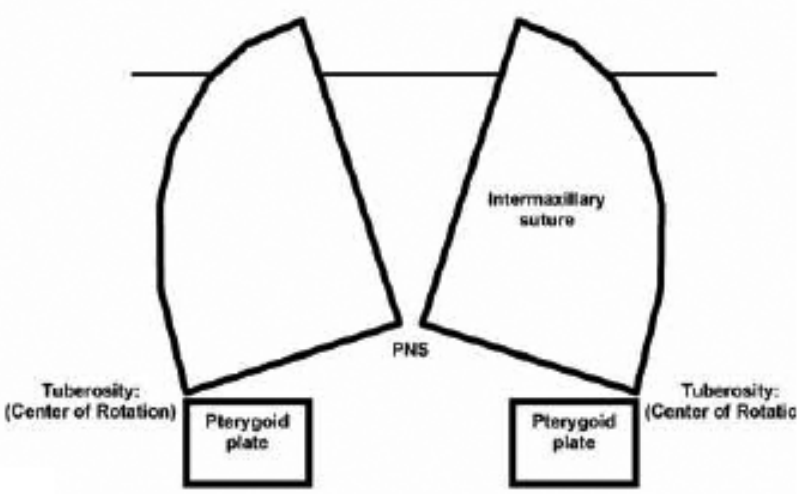




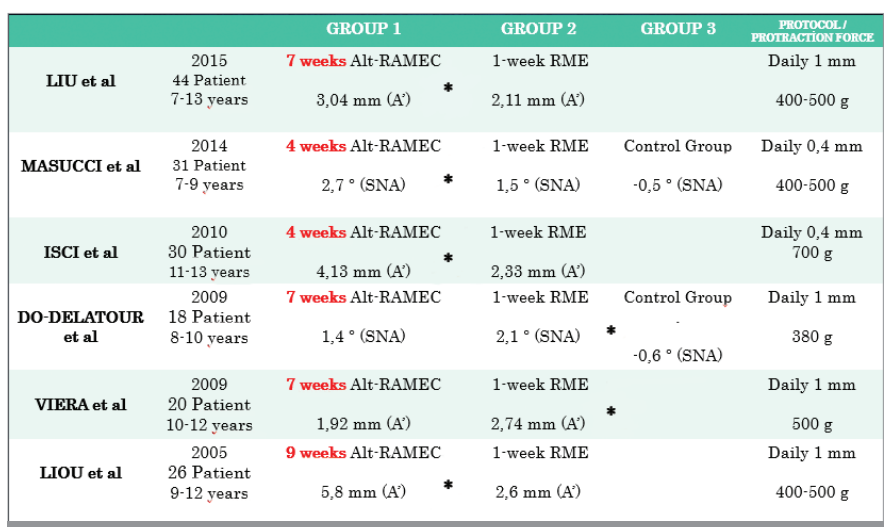

Figure 2. Studies comparing Alt-RAMEC protocol and RME

protocol (Figure 2). More forward movement was detected twice at the A point and maxilla in the groups involving the Alt-RAMEC protocol, except in two studies, than in the Alt-RAMEC protocol applied to the group.

Liou and Tsai (14) distinguished two groups of 26 patients with unilateral lip palate, ranging in age from 9 to 12 years, to investigate whether there was a difference between maxillary protraction after the Alt-RAMEC protocol and RME. RME was applied to the first group with normal hyrax screws for 1 week, whereas the Alt-RAMEC protocol was applied to the second group for 9 weeks at $1 \mathrm{~mm} /$ day with a double-hinged expander (Figure 3). Following this procedure, the researchers performed maxillary advancement for 6 months with the intraoral maxillary protraction springs in both groups. At the end of the study, more protraction was obtained in the Alt-RAMEC group (A point, 5.8 \pm 2.3 $\mathrm{mm}$ ) than in the RME group (A point, $2.6 \pm 1.5 \mathrm{~mm}$ ).

Both studies by Viera et al. (16) and Do-delatour et al. (17) reported more forward movement in the maxilla in the RME-treated group than most other studies. Do-delatour et al. (17), in their retrospective study, reported 18 individuals treated with the Alt-RAMEC protocol. They indicated that the Alt-RAMEC protocol alone is not sufficient in the maxillary procedure, and that the amount of protraction is higher in the RME-treated group than in the Alt-RAMEC-treated group. Viera et al. (16), in a study of 20 patients with unilateral lip palate, reported that the application of RME and Alt-RAMEC protocol does not make any difference before maxillary protraction, and that the effects are similar.

In 2010, Isci et al. (18) compared the dentofacial effects of the 4-week Alt-RAMEC protocol with the 1-week RME application prior to face mask in Class III patients in the growth and development period, where maxillary protraction was needed. Two groups of 15 individuals each were included in the study. At the end of the study, it was reported that the amount of movement of A point $(4.13 \mathrm{~mm})$ in the Alt-RAMEC group was twice as much in the RME group (2.33 $\mathrm{mm}$ ) (18).

Masucci et al. (19) performed face mask therapy with the 4-week Alt-RAMEC protocol for early treatment of Class III malocclusions and reported higher SNA and ANB angles and Wits values than face mask applied with normal RME.
In 2015, Liu et al. (20) compared face mask effects after the 7-week Alt-RAMEC protocol and RME. In the Alt-RAMEC group, there were more translations in the maxilla and less posterior rotation in the mandible.

Wilmes et al. (13) reported that face mask application with the Alt-RAMEC protocol is more effective in maxillary prolapse than conventional face mask therapy combined with normal RME as a result of implementing the 8-week Alt-RAMEC protocol with hyrax RME in two patients with Class III malocclusion.

Canturk et al. (21) compared the efficacy of using face mask during and after the 8-week Alt-RAMEC protocol for individuals with Class III malocclusion. The Alt-RAMEC protocol was not statistically different before or in combination with the face mask, but the maxillary showed significant prominence in both groups.

When experimental animal studies involving the Alt-RAMEC application were examined, Wang et al. (22), in a study in which they evaluated the opening of sutures on inbred cats in 2009, observed that the sagittal sutures are more open than with 1-week normal RME. It has also been reported that this protocol should be applied for $>5$ weeks for mobilization in coronal sutures of the maxilla.

Pithon et al. (23), in a systematic review, found that the Alt-RAMEC protocol is more effective than RME, that studies are inadequate with regard to relapse and recurrence, and that studies involving long-term outcomes are necessary.

\section{Relationship Between Skeletal Anchorage and Alt-RAMEC}

There are also studies in which two methods were used together to increase the skeletal effects of maxillary protraction. In 2011, Kaya et al. (24) used a face mask with miniplates placed on the lateral nasal wall of the maxillae following the 8-week Alt-RAMEC protocol, another method of increasing the skeletal effect of maxillary protraction in their studies. At the end of the study, they observed retraction in the mandibular incisors without movement in the maxillary incisor teeth, with a $2 \mathrm{~mm}$ maxillary advancement at an average of 9.9 months and a clockwise rotation of $0.8^{\circ}$ in the maxillae.

Implementation of Different Protocols in the Alt-RAMEC Protocol To the best of our knowledge, there is no study to date that indicates that Alt-RAMEC is a disadvantage or has a negative effect on tooth roots, alveolar bones, and periodontal tissues. RME has been the only method reported to promote root resorption in teeth as supported by some studies (25). It is important to determine the protocol that can provide the most effective protraction as soon as possible due to the risk of creating a jiggling effect (26), which occurs in recurring weeks with the Alt-RAMEC protocol.

There are a limited number of studies comparing different Alt-RAMEC protocols. Celikoglu et al. (27) examined skeletal and dentoalveolar changes after the 5-week and 9-week Alt-RAMEC protocols. The study has shown that the effects of the two protocols are similar. 

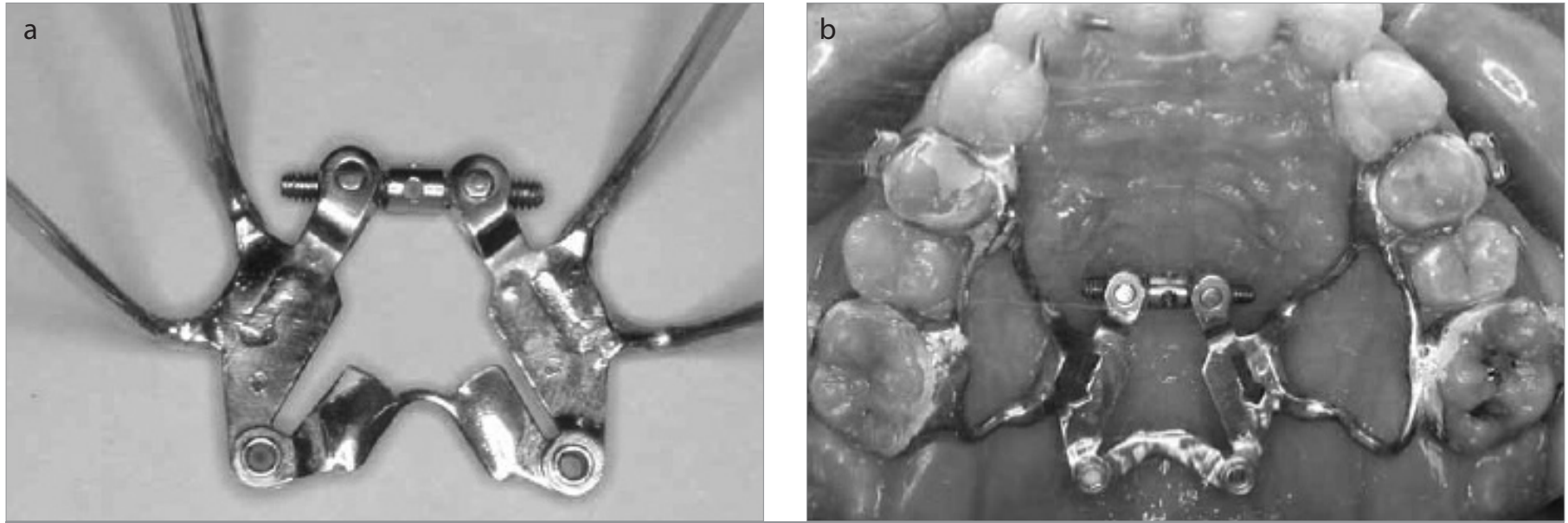

Figure 3. a, b. The configuration of a double-hinged rapid maxillary expander (12)

\section{Effect of Screw Type on Protocol}

When the Alt-RAMEC protocol was first introduced, it was applied with a double-hinged hyrax screw (Figure 3) as developed by Liou. In the studies conducted in the following years, this protocol was successfully applied with standard hyrax screws. However, to our knowledge, no study has investigated the effect of screw type on protocol or craniofacial and dentoalveolar construction.

However, in the study by Maino et al. (28) in 2018 with a different application, the hyrax screw was applied together with the hybrid anchorage. After applying 4 months of face masks, it was reported that dental effects were minimal.

\section{Influence of the Alt-RAMEC protocol on the airway}

Yılmaz et al. (29) evaluated airway measurements using cone beam computed tomography (CBCT) after 9 weeks of Alt-RAMEC protocol in an airway study. They reported that the A point moves forward, and that the upper airway and the nasal width increase.

Celikoglu et al. (30) examined the effect of maxillary protraction on the airway after different Alt-RAMEC protocols and reported that the effects of different protocols are similar.

In 2018, a study investigating the effect of the Alt-RAMEC protocol on the airway was also conducted using CBCT. The researchers compared individuals treated with RME and the Alt-RAMEC protocol in the study. They concluded in their study that the increase in nasal volume and nasopharyngeal volume was similar in both groups (31).

\section{Long-term Results}

The number of studies reporting the long-term results of the Alt-RAMEC protocol is rather limited. Although there are several studies on individuals with Class III malocclusion treated with maxillary protraction, to the best of our knowledge, there is no study that includes long-term results. The long-term studies performed on individuals with cleft lip and palate are both retrospective and relatively limited.
In 2018, 26 individuals with unilateral cleft lip and palate who had undergone the Alt-RAMEC protocol were subjected to 5-10year long-term follow-ups. As a result of the present study, it was reported that long-term results were stabilized when applied at the best time by providing sufficient protraction with a double-hinged expander (32).

\section{CONCLUSION}

Based on the literature review, the following conclusions were made:

- The application of the Alt-RAMEC protocol before maxillary protraction is an effective method for early treatment of patients with Class III malocclusion.

- In most of the studies, the Alt-RAMEC protocol appears to be more effective than RME.

- Further long-term studies on the Alt-RAMEC protocol are needed.

Peer-review: Externally peer-reviewed.

Conflict of Interest: The author has no conflict of interest to declare.

Financial Disclosure: The author declared that this study has received no financial support.

\section{REFERENCES}

1. Gürsoy N. Ortodontinin Biyolojik Temelleri, İstanbul: Doyuran 1988.

2. Gysel C. La progenie dans la famille de Dürer. Trans Europ Orthod Soc 1970; 55-60.

3. Litton SF, Ackerman LV, Isaacson J, Shapiro BL. A genetic study of Class III malocclusion. Am J Orthod 1970; 58: 565-77. [CrossRef]

4. Pancherz $\mathrm{H}$. Dentofacial orthopedics or orthognathic surgery: is it matter of age? Am J Orthod Dentofacial Orthop 2000; 117: 571-4. [CrossRef]

5. Ngan PW, Deguchi T, Roberts EW. Orthodontic Treatment of Class III Malocclusion. 2014.

6. Sung SJ, Baik HS. Assessment of skeletal and dental changes by maxillary protraction. Am J Orthod Dentofacial Orthop 1998; 114: 492-502. [CrossRef]

7. Nanda R. Protraction of maxilla in rhesus monkeys by controlled extraoral forces. Am J Orthod Dentofacial Orthop 1978; 74: 121-41. [CrossRef] 
8. Jackson GW, Kokich VG, Shapiro PA. Experimental and postexperimental response to anteriorly directed extraoral force in young Macaca nemestrina. Am J Orthod Dentofacial Orthop 1979; 75: 318-33. [CrossRef]

9. Baccetti T, Franchi L, McNamara JA. Treatment and posttreatment craniofacial changes after rapid maxillary expansion and facemask therapy. Am J Orthod Dentofacial Orthop 2000; 118: 404-13. [CrossRef]

10. Yu HS, Baik HS, Sung SJ, Kim KD, Cho YS. Three-dimensional finiteelement analysis of maxillary protraction with and without rapid palatal expansion. Eur J Orthod 2007; 29: 118-25. [CrossRef]

11. Sar C, Arman-Ozcirpici A, Uckan S, Yazici AC. Comparative evaluation of maxillary protraction with or without skeletal anchorage. Am J Orthod Dentofacial Orthop 2011; 139: 636-49. [CrossRef]

12. Liou EJ. Effective maxillary orthopedic protraction for growing Class III patients: a clinical application simulates distraction osteogenesis. Prog Orthod 2005; 6: 154-71.

13. Wilmes B, Ngan P, Liou EJ, Franchi L, Drescher D. Early class III facemask treatment with the hybrid hyrax and Alt-RAMEC protocol. J Clin Orthod 2014; 48: 84-93.

14. Liou EJ, Tsai WC. A new protocol for maxillary protraction in cleft patients: repetitive weekly protocol of alternate rapid maxillary expansions and constrictions. Cleft Palate Craniofac J 2005; 42: 21-7. [CrossRef]

15. Haas AJ. Long-term posttreatment evaluation of rapid palatal expansion. Angle Orthod 1980; 50: 189-217.

16. da Luz Vieira G, de Menezes LM, de Lima EM, Rizzatto S. Dentoskeletal Effects of Maxillary Protraction in Cleft Patients With Repetitive Weekly Protocol of Alternate Rapid Maxillary Expansions and Constrictions. Cleft Palate Craniofac J 2009; 46: 391-8. [CrossRef]

17. Do-de Latour TB, Ngan P, Martin CA, Razmus T, Gunel E. Effect of alternate maxillary expansion and contraction on protraction of the maxilla: A pilot study. Hong Kong Dent J 2009; 6: 72-82.

18. Isci $D$, Turk T, Elekdag-Turk S. Activation-deactivation rapid palatal expansion and reverse headgear in Class III cases. Eur J Orthod 2010; 32: 706-15. [CrossRef]

19. Masucci C, Franchi L, Giuntini V, Defraia E. Short-term effects of a modified Alt-RAMEC protocol for early treatment of Class III maloc- clusion: a controlled study. Orthod Craniofac Res 2014; 17: 259-69. [CrossRef]

20. Liu W, Zhou Y, Wang X, Liu D, Zhou S. Effect of maxillary protraction with alternating rapid palatal expansion and constriction vs expansion alone in maxillary retrusive patients: a single-center, randomized controlled trial. Am J Orthod Dentofacial Orthop 2015; 148: 641-51. [CrossRef]

21. Canturk BH, Celikoglu M. Comparison of the effects of face mask treatment started simultaneously and after the completion of the alternate rapid maxillary expansion and constriction procedure. Angle Orthod 2015; 85: 284-91. [CrossRef]

22. Wang YC, Chang PM, Liou EJ. Opening of circumaxillary sutures by alternate rapid maxillary expansions and constrictions. Angle Orthod 2009; 79: 230-4. [CrossRef]

23. Pithon MM, Santos NL, Santos CR, Baião FC, Pinheiro MC, Matos M Neto, et al. Is alternate rapid maxillary expansion and constriction an effective protocol in the treatment of Class III malocclusion? A systematic review. Dental Press J Orthod 2016; 21: 34-42. [CrossRef]

24. Kaya D, Kocadereli I, Kan B, Tasar F. Effects of facemask treatment anchored with miniplates after alternate rapid maxillary expansions and constrictions; a pilot study. Angle Orthod 2011; 81: 63946. [CrossRef]

25. Yilmaz BS, Kucukkeles N. Skeletal, soft tissue and airway changes following the alternate maxillary expansions and constrictions protocol. Angle Orthod 2014; 84: 868-77.

26. Celikoglu M, Buyukcavus MH. Changes in pharyngeal airway dimensions and hyoid bone position after maxillary protraction with different alternate rapid maxillary expansion and construction protocols: A prospective clinical study. Angle Orthod 2017; 87: 519-25. [CrossRef]

27. Vardimon AD, Graber TM, Voss LR, Lenke J. Determinants controlling iatrogenic external root resorption and repair during and after palatal expansion. Angle Orthod 1991; 61: 113-22.

28. Tosun Y. Sabit Ortodontik Apareylerin Biyomekanik Prensipleri. Ege Üniversitesi Basımevi; İzmir, 1999.

29. Celikoglu M, Buyukcavus MH. Evaluation of the effects of two different Alt-RAMEC procedures: Five weeks versus nine weeks. Aust Orthod J 2017; 33: 249-57. 